\title{
Apología Interdisciplinaria de la Metacognición del Aprendizaje Acelerado: discentes del área de Ciencias de la Educación y Medicina
}

\section{Interdisciplinary Apology of Accelerated Learning Metacognition: Learners in Education Sciences and Medicine}

\author{
DOI: $10.46932 / \mathrm{sfjdv2n4-005}$
}

Received in: March 1st, 2021

Accepted in: May 30th, 2021

\author{
Miryam Nava Cervantes \\ Lic. Pedagogía, Lic. Educación Preescolar, Maestría en Administración, Diplomado en Habientes \\ virtuales de aprendizaje, pasante de doctorado en Educación (línea de investigación Habilidades \\ sustentables en el SÉ-SER). \\ Institución actual: \\ Benemérita Universidad Autónoma de Puebla, México. Instituto de Estudios Universitarios, Puebla, \\ Mex. Normal Miguel Hidalgo, Puebla, Mex. \\ Domicilio \\ Priv. Jalisco \#413, El Carmen, PC 72000 Puebla, Pue. \\ Correo electrónico: minava456@gmail.com miryam.nava@correo.buap.mx
}

\section{RESUMEN}

La educación formal, informal y no formal están entrelazadas en la integridad de conocimientos del ser humano, en donde toda la sociedad global somos copartícipes de esa formación; hablar de educación es analizar un entramado de paradigmas que se convierten en nuevos retos para el docente del siglo XXI, por lo anterior, el presente trabajo está orientado al desarrollo interdisciplinario del área de estudio académico de discentes del segundo semestre de la carrera de medicina (Universidad pública) y del séptimo cuatrimestre de la Licenciatura de Ciencias de la Educación de la Universidad IEU (Instituto de Estudios Universitarios, institución particular), en donde se detectó con el trabajo diario en ambos grupos la necesidad de favorecer sus procesos metacognitivos, para mejorar su desempeño académico en su desarrollo cognitivo.

Teniendo como OBJETIVO favorecer el proceso metacognitivo en los discentes de medicina, por medio de la interdisciplinariedad con los estudiantes de ciencias de la educación con las técnicas del Aprendizaje Acelerado.

Palabras clave: aprendizaje acelerado, metacognición, cognición, interdisciplinariedad.

\begin{abstract}
Formal, informal and non-formal education are intertwined in the integrity of knowledge of the human being, where the whole global society is a co-participant of that formation; To talk about education is to analyze a framework of paradigms that become new challenges for the teacher of the XXI century, therefore, this work is oriented to the interdisciplinary development of the academic study area of students of the second semester of the career of medicine (public university) and the seventh quarter of the Bachelor of Science in Education of the IEU University (Institute of University Studies, a private institution), where it was detected with the aim of developing the academic study area of the students of the second semester of the career of medicine (public university) and the seventh quarter of the Bachelor of Science in Education of the IEU University (Institute of University Studies, This is a private institution), where it
\end{abstract}


was detected with the daily work in both groups the need to favor their metacognitive processes, to improve their academic performance in their cognitive development.

Having as OBJECTIVE to favor the metacognitive process in the medical students, through interdisciplinarity with the students of education sciences with the techniques of Accelerated Learning.

Key words: accelerated learning, metacognition, cognition, interdisciplinarity.

\section{DESARROLLO}

En este mundo globalizado interconectado con la comercialización, la política, la economía, entre otros, la educación no es la excepción, puesto que es la que mueve al mundo para favorecer los conocimientos del desarrollo humano en distintas áreas del conocimiento del ser, apoyándose de la interdisciplinariedad, multidisciplinariedad, transdisciplinariedad, abriendo el panorama en cualquier área de estudio formal e informal.

La propuesta se organizó en capacitar a los alumnos de 7mo cuatrimestre de la Licenciatura de Ciencias de la Educación dentro de la asignatura de Didáctica en la aplicación de las diferentes técnicas que ofrece el Aprendizaje Acelerado propuesto por Lozanov en los años setentas (sugestopedia) a los estudiantes del segundo semestre de la licenciatura de medicina, con la práctica de respiración relajada/sincronizada, ejercicios de gimnasia cerebral, programación neurolingüística, lectura rápida/lenta, subrayado de información relevante, resúmenes, escucha de música barroca, esquemas, mapas mentales o conceptuales, además de la visualización de contenidos; al aplicar dichas técnicas se vio como su desempeño académico mejoró significativamente dentro de clase; siendo más participativos, reflexivos, con buena actitud y relación con sus compañeros, por lo que se decide incluir esta forma de trabajo en el área de medicina, formulando la siguiente PREGUNTA DE INVESTIGACIÓN ¿Cómo las técnicas de aprendizaje acelerado favorecen el proceso metacognitivo, por medio de la interdisciplinariedad entre estudiantes de educación superior?

Lo anterior coadyuvó a trabajar de manera interdisciplinaria con los estudiantes de medicina, ya que se organizaron jornadas académicas dos veces por semana durante tres meses, en donde los discentes de Ciencias de la Educación asistieron a un grupo de medicina a orientarlos en el favorecimiento de su proceso metacognitivo con las técnicas del aprendizaje acelerado, que favorecerían la adquisión de sus conocimiento por medio de un pensamiento holístico, "acrecentando el proceso de enseñanza aprendizaje, en un ambiente favorable por medio de frecuencias, ritmos, tonos, lenguaje corporal, permitiendo un equilibrio entre los dos hemisferios cerebrales" (Sánchez y Andrade, 2003, p.201), poniendo juego la metacognición porque al unirlo con el aprendizaje acelerado se favoreció este proceso al recibir apoyo los estudiantes de medicina, al ser más receptivos y reflexivos de cómo estaban aprendiendo; como dice 
Martínez (2007, p.13) que es donde "se establece un control consciente y deliberado de las propias acciones cognitivas".

Afirmando que en todo proceso educativo debería estar presente el favorecimiento de la metacognición personal; en donde Carretero (2001) nos dice que es el "conocimiento que construyen las personas respecto del propio funcionamiento cognitivo", es la parte consciente de cómo comprendemos o entendemos algo, también Argüelles y Nagles (2007) "afirman que es el propio conocimiento de nosotros mismos (potencialidades personales) así como de las limitaciones cognitivas, que de alguna manera perturban el conocimiento, que en cuanto se comprende se puede ser activo en iniciar o dirigir su propio aprendizaje, dejando de ser reactivo ante estímulos", con las aportaciones anterior se pude mencionar que la interdisciplinariedad al estar vinculando la formación de estudiantes de dos carreras con perfiles distintos, se pueden apoyar entre sí, solucionando necesidades académica.

Pero, si los docentes fuéramos más conscientes de nuestro proceso metacognitivo, reaccionaríamos a favorecerlo en nuestros discentes, este proceso que los llevaría al éxito, no sólo académico sino personal, siendo estudiantes plenos en sus procesos del aprender aprender o en el aprender a desaprender en su vida diaria, favoreciendo el pensar en el razonamiento, procesamiento, retención del mismo conocimiento de sí mismo (Amestoy, 2010, p.11) y que es vínculo con los procesos cognitivos que favorecen a la mente en la creación y orden de la información que se recibe, en asimilar información de manera eficaz y eficiente facilitando los pensamientos; teniendo un papel fundamental los sentidos que ayudan a obtener información para procesarla cognitivamente y hacerla personal, destacando que serán diferentes aspectos del pensar que se estimularán.

Se destaca al autor Crespo (2002) que define la cognición “el conjunto de procesos mentales por medio de los cuales se organiza y se da sentido a la actividad, al pensamiento, al razonamiento, a la acción, y a diferentes formas de relación de las personas con el mundo y con la información que obtiene de él, siendo esta aportación magnifica para identificar lo que podemos fortalecer en nuestros estudiantes, pero también a través del tiempo se han dado diferentes aportaciones sobre teorías cognitivas, que coadyuvan a entender en la actualidad la importancia desarrollar la cognición y la metacognición en nuestra escuela de la vida para transformar nuestros entornos personales y sociales, yendo más allá de cuatro paredes de una institución educativa. Retomando que la palabra cognoscerse proviene del latín conocer en donde se entiende como conocimiento, proceso de conocer. Cognición son procesos mentales superiores de los seres humanos, que influyen la forma en que las personas conocen y comprenden el mundo, procesan la información, hacen juicios y toman decisiones, y describen su conocimiento y comprensión a los demás (Feldman, 2002, citado por Alonso, 2004), se identifica la vinculación de la cognición con la metacognición siendo un sujeto activo mentalmente, trascendiendo a otro nivel de pensamientos, 
reflejando la autonomía mental así como las emocionales. Ya que el proceso cognitivo, según Groomer (2005) considera los siguientes pasos para favorecer dicho proceso: Información recibida (INPUT) sensorialmente obteniendo Percepciones sobre la información recibida dando sentido a la información adquirida, teniendo como resultado un Almacenamiento de Aprendizaje y Memoria a largo plazo, para que cuando sea necesario se pueda Recuperar en la solución de alguna situación, dar información, actuar, aplicar en alguna práctica o incluso a adaptarse a otras circunstancias, en donde estará involucrado estrechamente el Pensamiento que pueden ser crítico o creativo, entre otros.

Otro de los tópicos centrados a analizar, es el Aprendizaje Acelerado propuesto por G. Lozanov en los años setenta (Bancroft, 1999), en donde asumió que el conocimiento se podía asimilar de diferente manera con la intención de obtener un conocimiento más eficaz, potencializando las capacidades de las personas, además de favorecer el proceso metacognitivo siendo más enriquecedor al ser más conscientes del autoaprendizaje, con la práctica de algunas técnicas como la escucha de música barroca, respiración sincronizada, ejercicios de coordinación manos, ojos, mente, (hemisferios) para ello se retoman los ejercicios de gimnasia cerebral propuesta por Ibarra ( 2000) en donde se destacan ejercicios para la relación, la concentración entre otros, además de complementar con un estado ALFA en donde la persona tiene que estar relaja para poder aprender con diferentes formas de adquirir un aprendizaje, como lo es la lectura rápida y lenta que debe ser eficaz para cualquier tipo de estudio, en donde se adquiere información, siendo una necesidad en esta era tecnológica, siendo un actividad intelectual cognitiva (García, 2000), en donde se tendrá que hacer una primera lectura rápida en el menor tiempo posible para favorecer una idea general de lo qué trata el texto, posteriormente una lectura lenta en la cual se realizará un subrayado de dos colores (rojo azul) de ideas que considere el lector que son importantes, posteriormente podrá elaborar un resumen y un organizador gráfico, que le facilitará recordar información relevante, resumiendo la información del texto original a un 50\%, además de que favorecerá el repaso del mismo contenido, para que cuando tenga el estudiante un examen sólo haga un repaso, cabe destacar que es la forma en la que se fue capacitando al grupo de 7mo. cuatrimestre de la Licenciatura en Ciencias de la Educción para que posteriormente fueran multiplicadores con los alumnos del 2do. semestre de la Licenciatura de medicina.

La aplicación de las técnicas mencionadas anteriormente se llevaron a cabo, como primer paso la relajación con la respiración y la música, un ejercicio de gimnasia cerebral, posteriormente realizar los tipos de lectura, resúmenes, organizadores gráficos (mapa mental, mapa conceptual, diagramas de flujo, esquemas, etcétera) recomendando que se realizaran a mano con colores, para que cada estudiante pidiera identificar cuál era la mejor forma en la que podían retener información significativamente y recordarla ante alguna situación, además de favorecer la interdisciplinariedad entre el área de Ciencias de la 
educación y la facultad de medicina, ya que estás se pueden apoyar significativamente para fortalecer en el ámbito personal como profesional.

Hay que destacar el papel que juega la interdisciplinariedad como elemento básico del presente trabajo; según Van de Linde (citado por Carvajal, 2010, p.4) como una estrategia pedagógica que implica la interacción de varias disciplinas entendidas como el diálogo y la colaboración para lograr la meta de un nuevo conocimiento, siendo que para Sotolorgo (2006) es el esfuerzo indagatorio y convergente entre varias disciplinas con el objetivo de saber a cerca de un objeto de estudio, entendiendo así la importancia de vincular dichas licenciaturas (Ciencias de la Educación y Medicina) para su apoyo metacognitivo. También resalta Flinterman, (2001) que explica que la “interdisciplinariedad es una colaboración de varias disciplinas, conceptos, metodologías o epistemologías e integradas explícitamente, en un resultado de enriquecimiento mutuo, esto hace, hoy en día que las diferentes áreas del conocimiento aporten entre ellas para fortalecer al mismo conocimiento en el logro de objetivos comunes abriendo otro panorama agrupando diversos saberes.

En cuanto a la multidisciplinariedad, se puede destacar el apoyo de diferentes ciencias sin perder su esencia para dar respuesta a una pregunta (pude ser de investigación), un proyecto multidisciplinar no conduce necesariamente a la interacción según Andrén, (2010), por eso es que se decide fortalecer en el presente trabajo basado en la Interdisciplinariedad, mientras que las transdisciplinariedad va más allá, trata de romper fronteras de las mismas ciencias, con una visión múltiple como de conocimientos múltiples (Nicolescu, 1998).

Se decidió proponer una METODOLOGÍA de tipo cualitativa en un estudio de casos, en donde se diseñaron y aplicaron algunas técnicas de recopilación de datos, como guía de observación, cuestionario (cerrado),entrevistas (semi-estructuradas) y un cuestionario abierto, lo anterior orientó la aplicación de una propuesta, teniendo como sujetos de estudio a 42 estudiantes.

Antes de iniciar la aplicación de la propuesta se aplicó un cuestionario a los estudiantes de medicina con 15 preguntas cerradas, en donde se tenían que responder sobre sus métodos de estudio, sus técnicas para investigar información, hábitos de estudio, alimentación, formas en la que mejor retenían información, los resultados fueron abrumadores al llegar a la conclusión de las respuestas arrojadas, que los alumnos en un $70 \%$ no tenían claro su forma de estudio el otro $30 \%$ consideraron que era leer información de libros, ver vídeos, y desconocían técnicas y métodos de estudio. En un 95\% carecía de un descanso de 8 hrs diarias para dormir, el $80 \%$ desconocía cuál era la mejor forma en la retenían información, mientras que el $20 \%$ lo hacían repasando diario sus apuntes. Un $70 \%$ estudiaban un día antes para el examen toda la tarde noche, sólo leyendo, el 20\% con resúmenes y un $10 \%$ con esquemas. Los 
resultados anteriores sirvieron para tener un diagnóstico más claro, ya que también presentaban bajo rendimiento académico dichos estudiantes.

Al poner en marcha la propuesta de la Apología Interdisciplinaria de la Metacognición del Aprendizaje Acelerado: discentes del área de Ciencias de la Educación y Medicina, se organizaron sesiones teóricas prácticas dos veces por semana durante tres meses, primero se capacitó a los estudiantes de la Licenciatura de Ciencias de la Educación (10 estudiantes) en las técnicas del Aprendizaje Acelerado y la metacognición para que llegaran a favorecer su propio proceso metacognitivo como capacitadores dentro de la materia de Didáctica que cursaban además de valorar los resultados de los estudiantes para poder iniciar el proyecto interdisciplinario, posteriormente se realizó una planeación didáctica de la capacitación que se le daría a los estudiantes de medicina (con objetivos, tiempo, actividades, materiales, instrumentos de evaluación) que coadyuvaría a la buena organización.

Al principio la mayoría de los alumnos se mostraron apáticos, pues no encontraban la practicidad de escuchas música o hacer esquemas, pero como fueron pasando los días de la práctica del taller con las actividades de reflexión, le fueron encontrando un significado, además de descubrirse ellos mismos, al identificar que cada uno tenía su propio estilo e aprendizaje, su forma de aprender y retener información de forma significativa, además de favorecer la empatía en el grupo y los estudiantes capacitadores del Aprendizaje Acelerado. Al terminar cada semana de práctica del taller se aplicaba una prueba cualitativa para analizar los avances del grupo así como entrevistas semiestructuradas con la finalidad de reforzar algún tema y una guía de observación de su trabajo en aula (una hora por sesión del taller). Cuando se finalizó el taller propuesto se obtuvieron los siguientes resultados generales por medio de un cuestionario cerrado (validado) en donde se destaca que el $90 \%$ ya identificaba su estilo de aprendizaje, cómo era su forma de aprender de manera significativa, el otro 10\% no logró tener resultados óptimos por situaciones personales como inasistencias al taller, problemas familiares. El 95\% realizaba lectura rápida y lenta para investigar temas de sus diferentes materias que cursaban, 5\% denotó que seguían con su misma forma de trabajo. El 92\% trabajó su metacognición para identificar cuál era la mejor forma en la podían estudiar y retener información, favoreciendo sus estrategias de estudio personales, como el uso de mapas mentales, esquemas, resúmenes con colores, esquemas, tarjetas de estudio, escuchar música, aplicación de gimnasia cerebral, descubriendo que podían potencializar su desempeño académico y su social con su grupo, el $8 \%$ arrojó que no se interesaron por desarrollar las técnicas de aprendizaje acelerado y por no asistir a todo el taller. En términos generales su pudo lograr el objetivo establecido y dar respuesta a la pregunta que al inicio se hizo, con la finalidad de potencializar a los estudiantes de licenciatura. 


\section{CONCLUSIONES}

Cada una de las técnicas del aprendizaje acelerado se fueron perfeccionando, por ejemplo, al realizar mapas conceptuales, ya que eran de mejor calidad en presentación y contenido, además de que expresaban comentarios sobre lo que leían cada semana.

Se puede decir que el Aprendizaje Acelerado, potencializa el desempeño del nivel superior, favoreciendo procesos metacognitivos y más si se trabaja entre pares en un proceso interdisciplinario.

La experiencia adquirida como docente durante la praxis del aprendizaje acelerado, tanto en la licenciatura de Ciencias de la educación como en la licenciatura en Medicina, comprueba cualitativamente y cuantitativamente que potencializa al estudiante universitario en su proceso metacognitivo, en un mejor desempeño académico, de habilidades como de actitudes siendo más reflexivo.

El trabajo académico entre pares, demuestra el enriquecimiento de habilidades, conocimientos, destrezas y actitudes personales como grupales.

Los estudiantes que practicaron constantemente las técnicas del aprendizaje acelerado, pudieron mejorar sus relaciones con el grupo, así como sus notas académicas.

En este mundo globalizado, tecnificado, recomiendo favorecer la interdisciplinariedad con habilidades blandas y duras, con la finalidad de favorecer a un conocimiento más sensible a las realidades que vivimos como docentes y estudiantes.

Todo docente debe buscar las estrategias idóneas para que sus alumnos desde edades muy tempranas favorezcan sus propios procesos metacognitivos.

El presente trabajo queda abierto para seguir haciendo aportaciones en pro de una formación más integral. 


\section{REFERENCIAS}

Argüelles D.N. (2007) Estrategias para comprender procesos de aprendizaje autónomo. Colombia. Alfaomega.

Alonso, A, (2004) La cognición, trabajos desarrollados, México.

Andrén, S. (2010). Un enfoque de investigación transdisciplinario, participativo y orientado a la acción. Universidad de Lund.

Amestoy, A. (2010) Habilidades básicas del pensamiento, México. Edi. Trillas.

Bancroft, W.J. (1999) Suggestopedia and Lenguage. Amsterdam: Routledge.

Carretero M. (2001) Enseñar a aprender. Introducción a la metacognición. Bilbao. Mensajero.

Carvajal, Y. (2010) Interdisciplinariedad: Desafíos para la educación superior y la investigación. Revista Luna Azul, Colombia

Crespo, A. (2002). Contemplando la cognición. En A. Crespo (Ed.), Cognició Humana. Mente, Ordenadores y Neuronas. Centro de estudios Ramón Areces Madrid. (pp. 27-35)

Flinterman, J. Teclemariam-Mesbah, R. \& Broerse JEW. (2001). Transdisciplinary: the new challenge for biomedical research. Bull Sci, 21:253-66.

García, C. (2000) Lectura rápida y comprensiva para todos). España, Narcea

Groome, D. (2005) An introduction to cognitive psychology. Processes and disorders.

Ibarra, L. (2000). Aprende mejor con Gimnasia Cerebral. España: GarniK ediciones

Lozanov G. (2978) Suggestology and Outlines of suggesto-pedy. Goordon and Breach Science Publisher. New York.

Novak J. Gorwin B. (1988) Mapas conceptuales para el aprendizaje significativo, en su aprendiendo a aprender, Barcelona. El campanario.

Nicolescu, B. La transdisciplinariedad, una nueva visión del mundo. Manifiesto. Paris: Ediciones Du Rocher. 1998.

Martínez (2007) La mediación en el proceso de aprendizaje, México, Edit. Bruño.

Sánchez G. Andrade E. (2004) Aprendizaje acelerado: un método para facilitar el aprendizaje, Instituto superior del estado de México, división Chalco. México. Editorial panorama.

Sotolorgo, P.L., Delgado C. J. (2006) La complejidad y el diálogo trasdisciplinario de saberes, Cap. IV, publicación la revolución contemporánea del saber y la complejidad social. 